\title{
Transport of a liquid water and methanol mixture through carbon nanotubes under a chemical potential gradient
}

\author{
Jie Zheng and Erin M. Lennon \\ Department of Chemical Engineering, University of Washington, Seattle, Washington 98195 \\ Heng-Kwong Tsao \\ Department of Chemical and Materials Engineering, National Central University, Chung-li, Taiwan 320, \\ Republic of China \\ Yu-Jane Sheng \\ Department of Chemical Engineering, National Taiwan University, Taipei, Taiwan 106, Republic of China
}

Shaoyi Jiang ${ }^{\text {a) }}$

Department of Chemical Engineering, University of Washington, Seattle, Washington 98195

(Received 27 January 2005; accepted 22 March 2005; published online 31 May 2005)

\begin{abstract}
In this work, we report a dual-control-volume grand canonical molecular dynamics simulation study of the transport of a water and methanol mixture under a fixed concentration gradient through nanotubes of various diameters and surface chemistries. Methanol and water are selected as fluid molecules since water represents a strongly polar molecule while methanol is intermediate between nonpolar and strongly polar molecules. Carboxyl acid $(-\mathrm{COOH})$ groups are anchored onto the inner wall of a carbon nanotube to alter the hydrophobic surface into a hydrophilic one. Results show that the transport of the mixture through hydrophilic tubes is faster than through hydrophobic nanotubes although the diffusion of the mixture is slower inside hydrophilic than hydrophobic pores due to a hydrogen network. Thus, the transport of the liquid mixture through the nanotubes is controlled by the pore entrance effect for which hydrogen bonding plays an important role. (C) 2005 American Institute of Physics. [DOI: 10.1063/1.1908619]
\end{abstract}

\section{INTRODUCTION}

Transport of fluids through nanoporous materials is currently a subject of great interest with many applications, such as molecule sieving, ${ }^{1}$ gas storage, ${ }^{2}$ molecular detection, ${ }^{3,4}$ and membrane separation. ${ }^{5,6}$ It is also important to the processes in biological transmembrane channels, ${ }^{7,8}$ such as gramicidin A and aquaporin (Ach). These biological channels are crucial to the transport of water molecules, ions, and other solutes across cells. These membrane channels vary in pore size and surface hydrophobicity. For example, the pore of the gramicidin $\mathrm{A}$ is formed by hydrophilic amino acids with a pore diameter of $\sim 4 \AA$ while the pore of the Ach channel is formed by hydrophobic side chains with a pore diameter of $\sim 12 \AA$. At present, little is known about the mechanism of selective ion/water diffusion through these biological pores. The single-walled nanotube (SWNT) can be used as a simple model to mimic biological ion channels since it has a uniform pore size and its surface chemistry can be altered. Several experimental studies ${ }^{9-11}$ show that the interior of nanotubes can be wetted and filled by liquids ${ }^{12,13}$ whose surface tension is less than about $200 \mathrm{mN} / \mathrm{m}$, such as water $(\sim 72 \mathrm{mN} / \mathrm{m})$ and most organic solvents $(<72 \mathrm{mN} / \mathrm{m})$. Beckstein and co-workers ${ }^{14,15}$ also found that a closed hydrophobic pore can be switched to an open state for water permeation by introducing the hydrophilic charac-

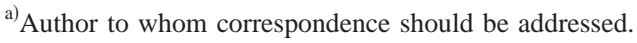

ter of the pore. However, it is still very challenging to perform experimental studies of the transport behavior of fluids through and within individual nanotube.

Molecular simulations are well suited to study the transport of fluids through nanopores. Allen and co-workers ${ }^{16}$ found from their molecular dynamics simulations that ion diffusion along the pore axis exhibits a general increase with channel radius in hydrophobic channels, but remains fairly low in hydrophilic channels. Hummer and co-workers ${ }^{17}$ found that small changes in the nanotube-water interactions $(\sim 0.05 \mathrm{kcal} / \mathrm{mol})$ can lead to large changes in water behavior between empty and filled states. This finding could be related to the gating mechanisms (close and open states) in biological ion channels. Kalra et al. ${ }^{18}$ performed long MD simulations $(\sim 180 \mathrm{~ns})$ to study the osmotic transport of water through nanotube membranes. It was concluded that the transport of water molecules was almost frictionless and governed by single-file diffusion mode, resulting from the formation of hydrogen-bond networks in nanotubes. However, none of the previous simulation studies of the transport of liquids through pores were performed under a fixed concentration gradient. Such a gradient is encountered in biological membrane channels where the fluid on either side of the membrane has a different concentration. It is desirable to understand how the transport of various fluids will be affected by pore geometry and surface chemistry under such conditions.

In this work, we study the transport of liquid mixtures 
through hydrophobic SWNTs or hydrophilic SWNTs anchored by $-\mathrm{COOH}$ groups under a fixed concentration gradient using dual-control-volume grand canonical molecular dynamics (DCV-GCMD) simulations. The DCV-GCMD simulation technique ${ }^{19-21}$ has been developed to study transport diffusion under a fixed concentration gradient. In DCV-GCMD simulations, adsorption/desorption, diffusion, and pore entrance effects can all be taken into account. However, to our knowledge, past DCV-GCMD simulations ${ }^{19-21}$ dealt exclusively with simple gases or gaseous mixtures through zeolites or other microporous materials. In this work, water and methanol are selected as model fluid molecules because water represents a strongly polar molecule while methanol is intermediate between nonpolar and strongly polar molecules. The interior of a SWNT can be modified by anchoring - $\mathrm{COOH}$ groups on the surface, changing the functionality of the carbon surface from hydrophobic to hydrophilic. In our previous DCV-GCMD simulations, ${ }^{22}$ we examined the transport of pure water and methanol through both hydrophobic and hydrophilic slit pores with a pore size of $23 \AA$ under a gradient. This work extends the previous one in two aspects: (a) hydrophobic and hydrophilic SWNTs of a cylindrical shape with smaller pore sizes are used to increase fluid confinement and (b) fluid mixtures are used to study the competitive transport of different fluids through different pores. The goal of this work is to provide a fundamental understanding of how fluid-fluid and fluid-surface interactions will affect the transport of a liquid mixture through a micropore.

\section{MODELS AND SIMULATION METHODOLOGY}

\section{A. Model systems}

A single-walled carbon nanotube can be viewed as a graphite sheet that is rolled up into a cylinder. As shown in Fig. 1, either the zig-zag $(n, 0)$ nanotube along $x$ axis or the armchair $(n, n)$ nanotube along $y$ axis can be formed, where the index $(n, m)$ indicates the helical structure of a nanotube. It was shown that the zig-zag or armchair structure of a nanotube has no effect on the diffusion behavior of molecular mixtures. ${ }^{23,24}$ In this study, we construct the armchair $(n, n)$ nanotube with an index $n=6-10$ corresponding to an inner diameter of $6.79,8.15,10.83,12.15$, or $13.57 \AA$. Unaltered SWNTs are hydrophobic. ${ }^{25}$ To prepare a hydrophilic nanotube, carboxyl acid groups $(-\mathrm{COOH})$ are first anchored on a flat graphite sheet with even spacing. The site density of $-\mathrm{COOH}$ groups on the surface is $1.59 / \mathrm{nm}^{2}$. Then, the graphite sheet with - $\mathrm{COOH}$ groups is rolled up and energy is minimized using the steepest descent method. These hydrophilic armchair nanotubes have the inner diameters of 7.33, 8.40,10.81, 12.22, and $13.41 \AA$ A respectively, which exclude the length of $-\mathrm{COOH}$ groups. Kyotani et al. ${ }^{26}$ proposed a template technique to chemically modify the inner wall of a carbon nanotube into a hydrophilic surface by $\mathrm{HNO}_{3}$ oxidation. The length of all SWNTs is $30.0 \AA$. The two ends of the SWNTs are open and $\mathrm{H}$ terminated. The universal force field was applied to describe the SWNTs. The carbon nanotubes used in this work are first optimized using molecular mechanics and then kept rigid during the simulations.
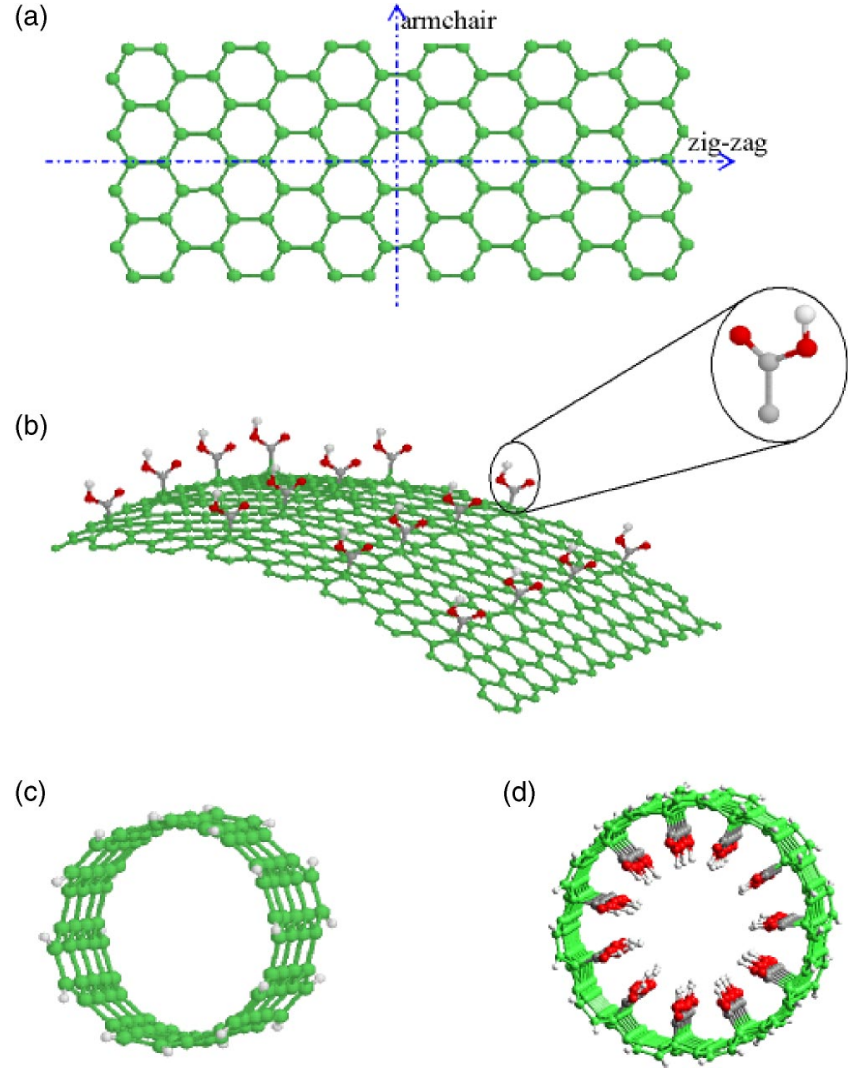

FIG. 1. (a) A hexagonal graphite sheet to create a zig-zag or armchair nanotube by rolling up along $x$ or $y$ axis; (b) A graphite sheet with active - $\mathrm{COOH}$ groups; (c) A armchair SWNT with a diameter of $8.15 \AA$; (d) A hydrophilic SWNT decorated with $-\mathrm{COOH}$ groups having an inner diameter of $8.40 \AA$.

A binary mixture of water and methanol is examined in this study. We use the simple point charge (SPC) model of Berendsen et al $^{27}$ to describe water-water interactions. The SPC model gives reasonable structural and thermodynamic properties, such as liquid density, heat of vaporization, and diffusion constant as compared with experimental data. ${ }^{27}$ For methanol, a number of simple models have been previously proposed. The models proposed by Jorgensen $e t a{ }^{28}$ and by Haughney et $a .^{29}$ have been widely used for liquid simulations. Both models give results for a range of properties that are in good agreement with the available experimental values. In our simulations, each methanol molecule is described by the model for optimized potentials for liquid simulations (OPLS) proposed by Jorgensen et al. ${ }^{28}$ because Jorgensen's model can reproduce both gas-phase dimmer properties and liquid density at ambient temperature and pressure. In the OPLS model, three interaction sites are positioned on the oxygen $(\mathrm{O})$ nuclei, hydroxyl proton $(\mathrm{H})$, and united methyl $\left(\mathrm{CH}_{3}\right)$ group centered on the carbon $(\mathrm{C})$, respectively.

Fluid-nanotube and fluid-fluid interactions are given by the sum of the long-range Coulombic and short-range van der Waals (VDW) terms,

$$
U_{f f}=\sum_{i}^{n} \sum_{j}^{n}\left\{\frac{q_{i} q_{j}}{4 \pi \varepsilon_{0} r_{i j}}+4 \varepsilon_{i j}\left[\left(\frac{\sigma_{i j}}{r_{i j}}\right)^{12}-\left(\frac{\sigma_{i j}}{r_{i j}}\right)^{6}\right]\right\} .
$$

where $r_{i j}$ is the separation distance between the atoms $i$ and $j, q_{i}$ is the partial charge of atom $i, \sigma_{i j}$ is the atom collision 
TABLE I. The LJ parameters and partial charges for water, methanol, $-\mathrm{COOH}$ group, and nanotube.

\begin{tabular}{|c|c|c|c|c|}
\hline & Site & $\sigma(\mathrm{nm})$ & $\varepsilon(\mathrm{kJ} / \mathrm{mol})$ & $q(\mathrm{e})$ \\
\hline \multicolumn{5}{|l|}{$\mathrm{H}_{2} \mathrm{O}$} \\
\hline & $\mathrm{O}$ & 0.31656 & 0.1554 & -0.82 \\
\hline & $\mathrm{H}$ & 0.0 & 0.0 & 0.41 \\
\hline \multicolumn{5}{|l|}{$\mathrm{CH}_{3} \mathrm{OH}$} \\
\hline & $\mathrm{CH}_{3}$ & 0.3775 & 0.8661 & 0.265 \\
\hline & $\mathrm{O}$ & 0.3070 & 0.7113 & -0.7 \\
\hline & $\mathrm{H}$ & 0.0 & 0.0 & 0.435 \\
\hline \multicolumn{5}{|l|}{$-\mathrm{COOH}$} \\
\hline & $\mathrm{C}^{\mathrm{a}}$ & 0.34 & 0.2328 & 0.08 \\
\hline & $\mathrm{C}$ & 0.375 & 0.4393 & 0.55 \\
\hline & $=\mathrm{O}$ & 0.296 & 0.8786 & -0.50 \\
\hline & $\mathrm{O}$ & 0.30 & 0.7133 & -0.58 \\
\hline & $\mathrm{H}$ & 0.0 & 0.0 & 0.45 \\
\hline \multicolumn{5}{|l|}{ Nanotube } \\
\hline & $\mathrm{C}$ & 0.34 & 0.2328 & 0.0 \\
\hline
\end{tabular}

${ }^{\mathrm{a}}$ Anchored carbon on the nanotube.

diameter, and $\varepsilon_{i j}$ is the well depth. The Lennard-Jones (LJ) parameters are calculated using the Lorentz-Berthelot combining rule, where $\sigma_{i j}=\left(\sigma_{i}+\sigma_{j}\right) / 2$ and $\varepsilon_{i j}=\sqrt{\varepsilon_{i} \varepsilon_{j}}$. Table I lists the LJ parameters and partial charges used in this study.

\section{B. Simulation methodology}

In this work, the DCV-GCMD technique is employed to study the transport of molecules from liquid phase to vacuum under a fixed chemical potential gradient. The DCV-GCMD technique $^{19-22}$ is a combination of grand canonical Monte Carlo (GCMC) and molecular dynamics simulations. The simulation system of $8.6 \times 2.4 \times 2.4 \mathrm{~nm}^{3}$ is divided into two control volumes (CV1 and CV2), two buffer boxes (B1 and B2), and a flow region, as shown in Fig. 2. The CV1 and CV2 contain bulk fluids at high and low chemical potentials, respectively. The simulation box is confined by two hard walls, located at each end of the box in the $x$ direction. The SWNT is placed in the middle of the simulation box (i.e., the flow region). A typical DCV-GCMD simulation is performed following the procedure below.

Initial condition. The initial configuration of the watermethanol mixture $(64 \mathrm{~mol} \%-36 \mathrm{~mol} \%)$ was first generated and equilibrated by a separate GCMC simulation in the bulk with dimensions of $2.4 \times 2.4 \times 2.4 \mathrm{~nm}^{3}$. Then, the preequilibrated box of the fluid mixture is placed in the CV1. The use of vacuum in the sink (CV2) eliminates the need to specify the final product composition of a mixture. The buffer boxes of B1 and B2 are left empty at the beginning of simulations so that entrance (or exit) effects can be investigated.

GCMC phase. During GCMC $(\mu V T)$ simulations, the chemical potential of each component in the CV1 is maintained constant by carrying out a sufficient number of GCMC trials so that the source (CV1) contains a liquid mixture with a fixed composition $(64 \mathrm{~mol} \%-36 \mathrm{~mol} \%)$ while the sink (CV2) is kept under a vacuum. In GCMC simulations, there are four types of trials, i.e., move (translation and rotation), insertion, deletion, and swap using the Metropolis algorithm. For a translation or rotation movement, a trial configuration is generated by randomly choosing one molecule in the CV1 and giving it either a uniform random displacement or a rotation about a random axis. The displacement of each move is adjusted to ensure an acceptance ratio of $\sim 50 \%$. For an insertion trial, the position and orientation of an individual molecule are chosen at random and the molecule is then inserted. The velocity of the new created molecule is assigned with a Maxwell-Bolzmann distribution at $300 \mathrm{~K}$. For a destruction trial, a chosen molecule is removed from the system. In a binary mixture system, the swap technique (i.e., changing the identity of two species) has been shown to increase the speed of convergence and reduce fluctuations in particle number once equilibrium is reached.

$M D$ phase. After a set number of GCMC steps in the control volumes, the canonical ensemble ( $N V T)$ MD simulation is performed to move all liquid molecules (water and methanol) in the entire simulation box. Since bond vibrations are very fast, any covalent bonds involving hydrogen atoms in water and methanol are kept rigid using the RATTLE algorithm with a geometric tolerance of 0.0001 . Newtonian equations of motion are integrated using the velocity Verlet algorithm with a time step of $1.0 \mathrm{fs}$. The system is coupled to a bath at a temperature of $300 \mathrm{~K}$ using the velocity scaling algorithm.

The liquid mixture flows from the CV1 (liquid reservoir) to the buffer zone, before entering the SWNT and finally the CV2 (vacuum sink). As the MD simulation progresses the number of particles in the CV1 decreases. The chemical potential of each component in the control volumes changes accordingly. Thus, GCMC simulations are periodically performed in each control volume until the chemical potential is restored to its prescribed value. In this work, an optimum value for $n_{\mathrm{GCMC}} / n_{\mathrm{MD}}$ (the ratio of stochastic to dynamic steps) of 100-150 is used to yield the correct concentration of the water and methanol mixture and keep a minimum computing time. The periodic boundary conditions and minimum image conventions are only applied to the $y$ and $z$ directions for fluid molecules in the control volumes and the

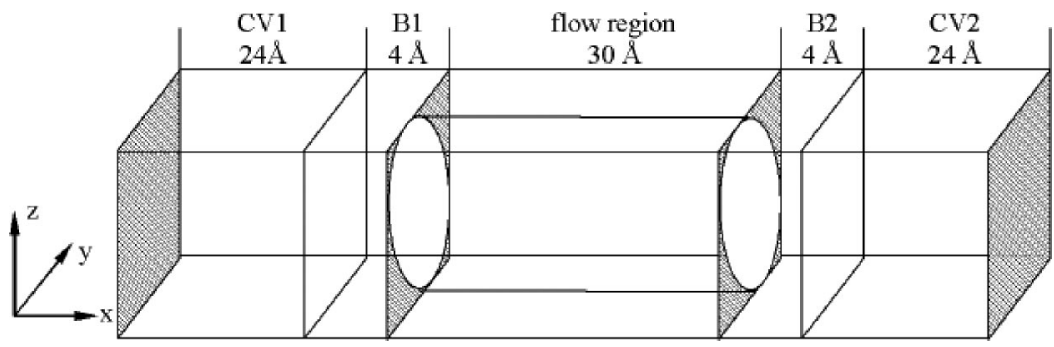

FIG. 2. Schematic representation of a DCV-GCMD simulation cell. MD simulations are performed for the entire system while GCMC simulations are performed in between MD simulations to maintain the chemical potentials of both control volumes. 
(a)
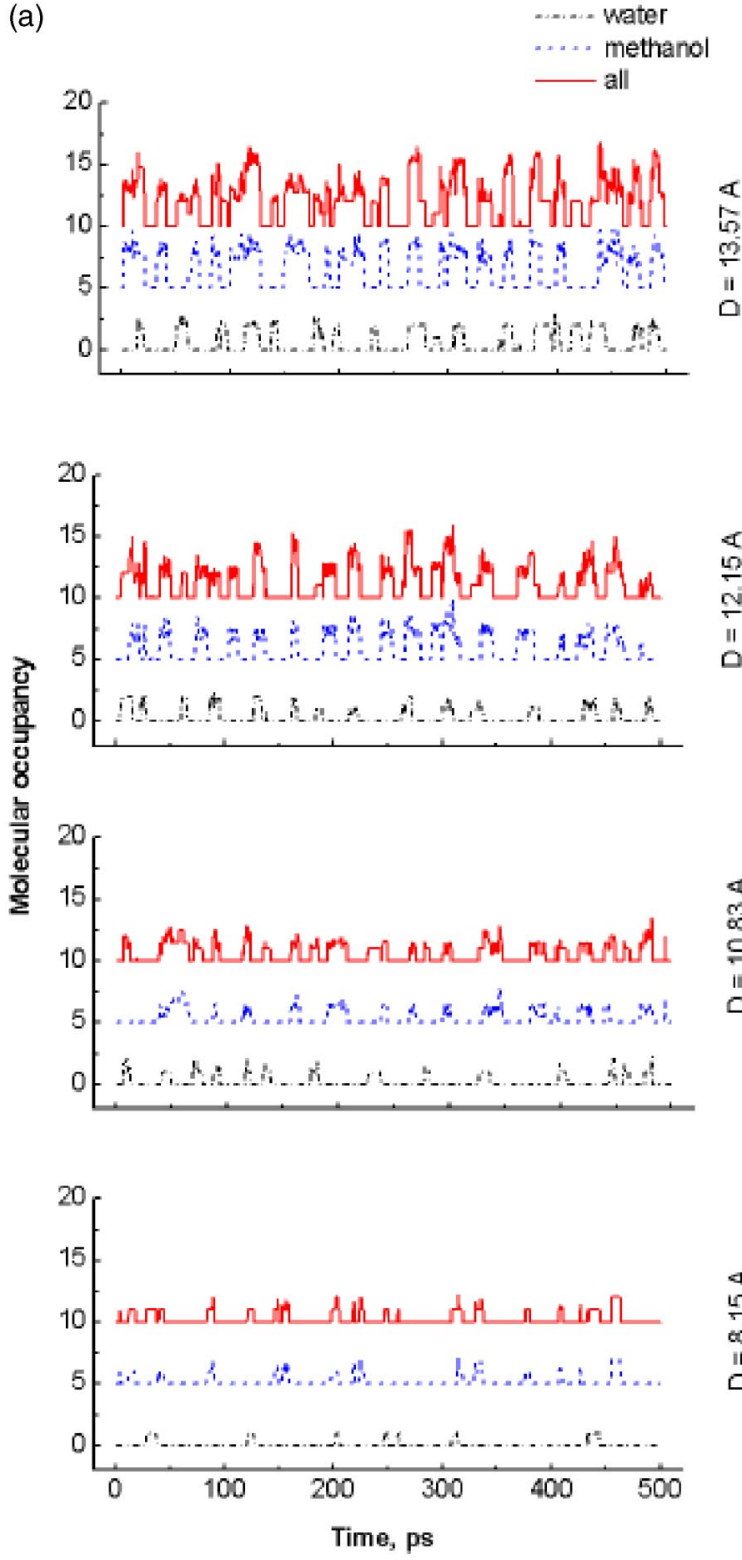

(b)
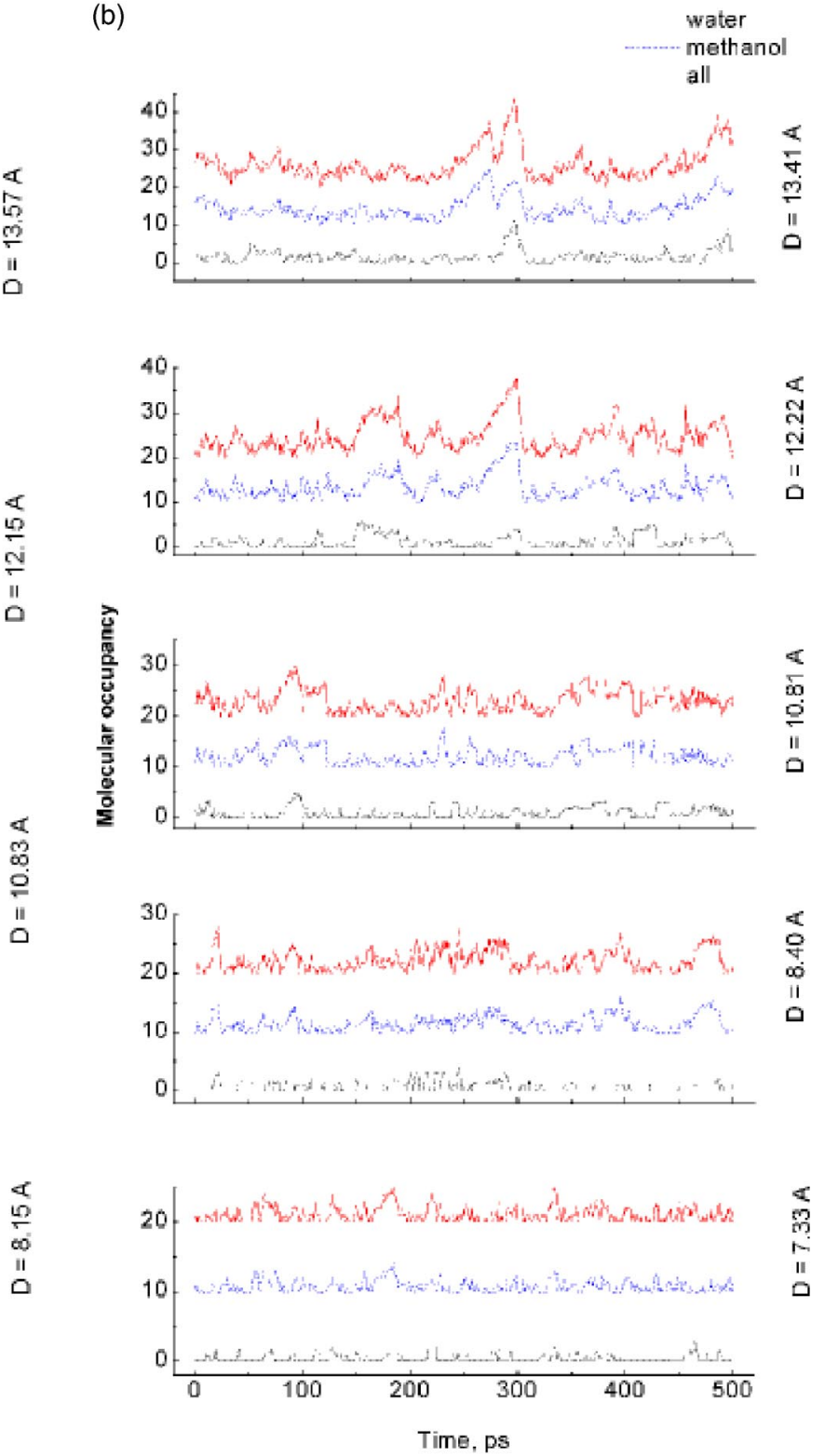

FIG. 3. Occupancy profiles of water and methanol molecules inside a hydrophobic (a) or hydrophilic (b) nanotube as a function of time. For hydrophobic nanotubes, the curves of methanol molecules and total molecules are shifted by 5 and 10, respectively. For hydrophilic nanotubes, the curves of methanol molecules and total molecules are shifted by 10 and 20 , respectively.

buffer boxes. The nonbonded interactions are described by the cut-shifted potential at a cutoff of $1.0 \mathrm{~nm}$ for short-range VDW interactions and $1.2 \mathrm{~nm}$ for long-range Coulombic interactions. All simulations are run for 3-5 ns to reach steady state. The properties reported are collected during the last 1 ns unless specified otherwise.

\section{RESULTS AND DISCUSSION}

Figure 3 shows the occupancy profile of water and methanol molecules as a function of time for both hydrophobic and hydrophilic SWNTs at several pore sizes. The number of water and methanol molecules inside the nanotube is graphed for the last 500 ps of each simulation. For the hydrophobic SWNTs, the occupancy of water and methanol molecules increases with the size of the nanotubes. The smallest $(6,6)$ SWNT shows that neither water nor methanol can enter the nanotube. For other hydrophobic nanotubes with diameters $>6.79 \AA$, the occupancy of water and methanol molecules oscillates between empty and partially filled states. The water and methanol mixture flows through the hydrophobic nanotubes in a pulselike fashion. Similar oscillating behavior was also observed by others in both experiments $^{30}$ and simulations. ${ }^{14,17}$ Unlike the hydrophobic SWNTs, the occupancy profiles in Fig. 3(b) show that the water and methanol mixture diffuses through the hydrophilic nanotubes in a continuous fashion. The number of adsorbed water and methanol molecules in the hydrophilic nanotubes increases with their size. Beckstein and co-workers ${ }^{14,15}$ found that a closed hydrophobic pore can be switched to an open state for water permeation by introducing the hydrophilic character of the pore. It should be noted that, for the narrowest hydrophilic nanotube with similar diameter to the 
(a)

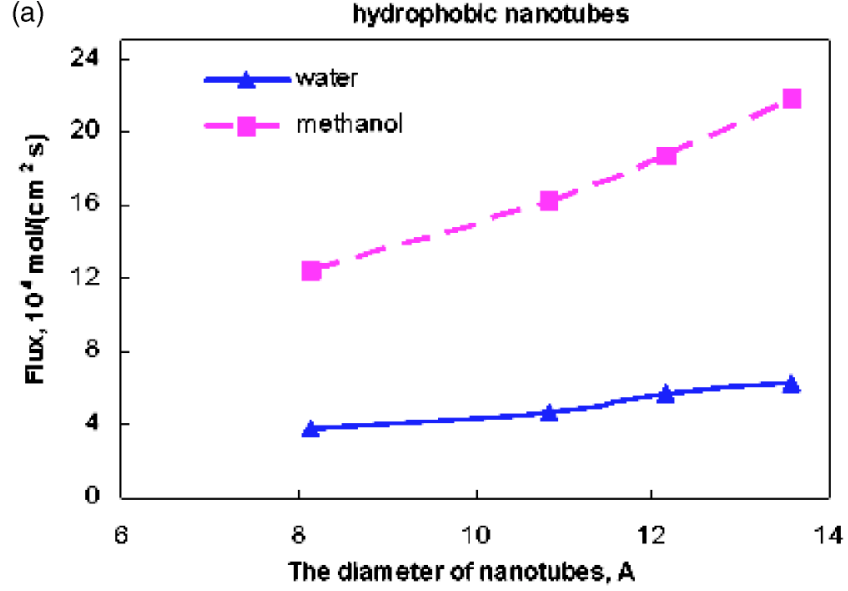

(b) hydrophilic nanotubes

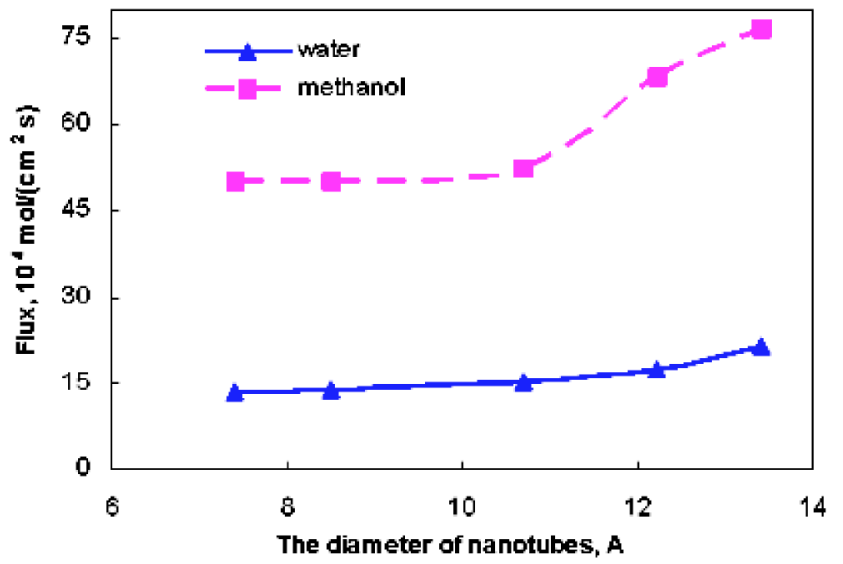

FIG. 4. Fluxes of water and methanol molecules as a function of the diameters of a hydrophobic (a) or hydrophilic (b) nanotube. The lines are drawn to guide eyes.

hydrophobic $(6,6)$ tube, both water and methanol molecules can enter the pore while this phenomena is not observed for the hydrophobic $(6,6)$ tube.

To examine the effect of confinement on the molecular motion of liquid water and methanol inside the nanotubes, we calculated the flux by measuring the net movement of each component crossing a given plane in the steady state. Invariance of the fluxes with time indicates that the system reaches steady state. The flux can be expressed as

$$
J_{i}=\frac{N_{i}^{\mathrm{LR}}-N_{i}^{\mathrm{RL}}}{n_{\text {steps }} \Delta t A_{y z}},
$$

where $N_{i}^{\mathrm{LR}}$ and $N_{i}^{\mathrm{RL}}$ are the number of the fluid molecules of component $i$ moving from the left to the right and vice versa, respectively; $n_{\text {steps }}$ is the number of MD steps; $A_{y z}$ is the area of the $y z$ plane; and $\Delta t$ is the MD time step of $1 \mathrm{fs}$. Figure 4 shows the fluxes of water and methanol as a function of time for SWNTs with different pore diameters. Simulation results show that the fluxes of water and methanol increase with increasing the diameter of the nanotube. The fluxes of water and methanol are significantly higher in the hydrophilic nanotubes than in the hydrophobic nanotubes.

Transport of fluid molecules through a nanotube is composed of two consecutive steps: (a) entering the nanotube from the bulk (i.e., pore entrance effect) and (b) flowing

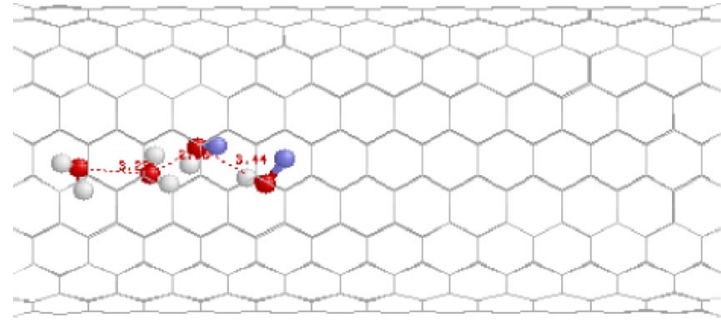

FIG. 5. Snapshot of a hydrophobic $(10,10)$ nanotube containing water and methanol molecules. Hydrogen bonds are marked by dashed lines with their distances. The color scheme is oxygen in red, hydrogen in white, and united $\mathrm{CH}_{3}$ in blue.

through the nanotube (i.e., adsorption/desorption and diffusion inside the pore). The compilation of these two steps results in a net flux. In the first step, the entry of fluid molecules into a nanotube can be described as follows. For a hydrophobic nanotube, the first molecule initially enters an empty nanotube from the bulk when driven by a concentration gradient and thermal fluctuations. Subsequently, more molecules in the bulk are dragged into the tube by forming a continuous chain with those inside the pore through hydrogen bonding, ${ }^{31}$ as shown in Fig. 5. Similar single-file arrangements of liquid molecules were also observed in other simulation studies of water confined in hydrophobic nanotubes. ${ }^{18,32,33}$ When fluid molecules enter a nanopore, some hydrogen bonds present in the bulk phase could be lost due to confinement. Hummer et al. ${ }^{17}$ recently reported that water molecules could enter a hydrophobic nanotube from the bulk, although it was expected that the hydrophobic pore had the tendency to discourage the passage of water molecules due to its water-repellent properties and its limited interior space. They found that water molecules entering a hydrophobic nanotube from the bulk lose two out of four hydrogen bonds, but a fraction of lost energy was recovered through attractive water-nanotube VDW interactions. For hydrophilic pores, as compared with hydrophobic nanotubes, the energy expense of breaking the network of hydrogen bonds in the bulk of fluids can be greatly compensated by attractive interactions between fluid molecules and $-\mathrm{COOH}$ groups near the entrance of the hydrophilic nanotubes. Thus, more water and methanol molecules are able to enter and wet the hydrophilic SWNTs (Fig. 3). In the second step, the adsorption/desorption and diffusion of fluid molecules inside a nanotube are governed by fluid-fluid and fluid-surface interactions inside the pore, particularly for the hydrogen bonding in the systems studied here. In Table II, the average number of hydrogen bonds (HB) per molecule $\left\langle n_{\mathrm{HB}}\right\rangle$ is reported in each nanotube for methanol and water calculated according to a geometrical definition. A hydrogen bond exists if the donor-acceptor distance is less than $0.35 \mathrm{~nm}$ and the hydrogen donor-acceptor angle is smaller than $60^{\circ}$. As can be seen in Table II, $\left\langle n_{\mathrm{HB}}\right\rangle$ of both water and methanol is higher in the hydrophilic nanotubes than in the hydrophobic nanotubes. The formation of a hydrogen-bond network among fluid molecules and between fluid molecules and the surface $-\mathrm{COOH}$ groups can greatly suppress the mobility of fluid molecules inside the hydrophilic nanotubes. It should be pointed out that the number of hydrogen bonds strongly 
TABLE II. The average number of hydrogen bonds per molecule $\left\langle n_{\mathrm{HB}}\right\rangle$ in both hydrophobic and hydrophilic nanotubes.

\begin{tabular}{cccccccc}
\hline \hline \multicolumn{3}{c}{ Hydrophobic nanotube } & & & \multicolumn{3}{c}{ Hydrophilic nanotube } \\
\cline { 1 - 1 } \cline { 5 - 7 } Diameter $(\AA)$ & Water & Methanol & & Diameter $(\AA)$ & Water & Methanol \\
\hline \multirow{2}{*}{6.79} & $/$ & $/$ & & 7.33 & 2.85 & 2.13 \\
8.15 & 0 & 0 & & 8.40 & 2.96 & 1.80 \\
10.83 & 0 & 0 & & 10.81 & 1.74 & 1.20 \\
12.15 & 0.8 & 0.5 & & 12.22 & 1.40 & 0.75 \\
13.57 & 1.0 & 0.4 & & 13.41 & 1.32 & 0.71 \\
\hline \hline
\end{tabular}

depends on the diameter of a nanotube. For the hydrophobic nanotubes, the liquid mixture does not form a complete hydrogen-bond network due to a limited number of adsorbed molecules inside the pore. For the hydrophilic tubes, the average number of hydrogen bonds for both water and methanol increases with a decrease in the diameter of the nanotubes. Fluid molecules are more likely to form hydrogen bonds with either themselves or with the hydrophilic surfaces inside the smaller nanotubes due to geometrical confinement. However, the density of the fluid mixture is still quite low inside the nanotubes as compared to that in the bulk. To further examine the effect of hydrogen bonding on molecular mobility, we performed additional MD simulations of a single molecule in both hydrophobic and hydrophilic nanotubes. Simulation results show that the selfdiffusion coefficients of both water and methanol molecules in the hydrophilic nanotubes $\left(10^{-7} \mathrm{~cm}^{2} / \mathrm{s}\right)$ are two orders of magnitude smaller than in the hydrophobic nanotubes $\left(10^{-5} \mathrm{~cm}^{2} / \mathrm{s}\right)$. Hydrogen bonds slow down molecular motion. The transport of fluids inside hydrophobic nanotubes is faster than inside hydrophilic nanotubes. Allen and co-workers ${ }^{16}$ also found from their molecular dynamics simulations that ion diffusion along the pore axis exhibits a general increase with channel radius in hydrophobic channels, but remains fairly low in hydrophilic channels. Thus, for a hydrophobic nanotube, it is difficult for water and methanol molecules to enter the pore, but they will quickly diffuse through it once inside the pore. On the contrary, for a hydrophilic nanotube, water and methanol molecules easily enter the pore but have a difficult time flowing through it. The net effect is that fluid transport through hydrophilic nanotubes is faster than through hydrophobic ones. This indicates that fluid transport across the pore is controlled by the entrance effects. However, if the nanotube has a larger diameter and is sufficiently long, then the pore entrance effect will weaken with respect to the effect due to fluid adsorption/desorption and diffusion inside the pore.

For the transport of the liquid mixture, it is interesting to note the selectivity of the pores for the methanol or water mixtures. The separation efficiency for component $j$ over component $i$ to transport through a pore can be described by

$$
S_{i / j}=\frac{J_{j} / J_{i}}{x_{j} / x_{i}},
$$

where $J_{j} / J_{i}$ is a ratio of flux of component $j$ to component $i$, and $x_{j} / x_{i}$ is a ratio of the molar fraction of component $j$ to that of component $i$. Table III shows that the separation effi- ciency $S_{\text {methanol/water }}$ is $\sim 6.0$ and 6.5 for hydrophobic and hydrophilic nanotubes, respectively. For the transport of a liquid mixture through a pore, each component has different entrance effects and behaviors inside the pore. For example, for the methanol and water mixture, water is a strong hydrogen-bonded polar molecule while methanol is intermediate between nonpolar and strongly polar molecules. In the bulk phase, liquid water and methanol have an average number of hydrogen bonds of 3.57 and 2.0, respectively. ${ }^{34}$ Thus, methanol molecules are easier to enter the mouth of a nanotube than water molecules for both hydrophobic and hydrophilic pores due to smaller loss in energy because of confinement. Methanol is transported faster inside the hydrophobic pore than the hydrophilic pore due to weaker methanolsurface hydrogen bonding. Similar selectivity can also be achieved by means of conventional VLE operation. ${ }^{35}$ The vapor pressures of water and methanol at room temperature are 3.1691 and $16.851 \mathrm{kPa}$, respectively. Their activity coefficients calculated from the Wilson equation are 1.072 for water and 1.188 for methanol, respectively. Based on vaporliquid equilibrium calculations,

$$
\begin{aligned}
\frac{y_{\text {methanol }} / y_{\text {water }}}{x_{\text {methanol }} / x_{\text {water }}} & =\frac{P_{\text {methanol }}^{0} \gamma_{\text {methanol }}}{P_{\text {water }}^{0} \gamma_{\text {water }}} \\
& =\frac{16.851 \times 1.188}{3.1691 \times 1.072}=5.89 .
\end{aligned}
$$

However, by adjusting operating conditions (e.g., temperature and feed composition) or pore parameters (e.g., pore size and surface property), the selective separation of water and alcohol mixtures can be achieved. Further studies are needed to address this issue.

TABLE III. Selectivity of methanol over water in both hydrophobic and hydrophilic nanotubes.

\begin{tabular}{ccccc}
\hline \hline \multicolumn{2}{c}{ Hydrophobic nanotubes } & & \multicolumn{2}{c}{ Hydrophilic nanotubes } \\
\cline { 1 - 2 } \cline { 5 - 5 } $\begin{array}{c}\text { Diameter } \\
(\AA)\end{array}$ & $\begin{array}{c}\text { Selectivity } \\
\left(S_{\text {methanol/water })}\right.\end{array}$ & & $\begin{array}{c}\text { Diameter } \\
(\AA)\end{array}$ & $\begin{array}{c}\text { Selectivity } \\
\left(S_{\text {methanol/water }}\right)\end{array}$ \\
\hline 6.79 & $/$ & & 7.33 & 6.62 \\
8.15 & 5.79 & & 8.40 & 6.48 \\
10.83 & 6.17 & & 10.81 & 6.10 \\
12.15 & 5.84 & & 12.22 & 6.92 \\
13.57 & 6.16 & & 13.41 & 6.40 \\
\hline \hline
\end{tabular}




\section{CONCLUSIONS}

In this work, DCV-GCMD simulations were performed to study the transport of a liquid mixture of water and methanol through hydrophobic and hydrophilic nanotubes of varying diameters under a chemical potential gradient. For the hydrophobic nanotubes, water and methanol molecules are difficult to enter the pore, but will quickly diffuse through the pore once they enter the pore. On the contrary, for the hydrophilic nanotubes, water and methanol molecules easily enter the pore, but difficult to flow through the pores. The net effect is that fluid transport through hydrophilic tubes is faster than through hydrophobic ones. Results indicate that fluid transport across the pores is controlled by the pore entrance effects. It is observed that the water and methanol mixture flows through the hydrophobic nanotubes in a pulse fashion while through the hydrophilic nanotubes in a continuous fashion. It appears that similar selectivity can also be achieved by means of conventional VLE operation. However, it is expected that the pores can provide more desirable selectivity when appropriate conditions are adopted.

\section{ACKNOWLEDGMENTS}

The authors thank Professor Bruce A. Finlayson for helpful discussions. They gratefully acknowledge the National Science Foundation (Grant No. CTS-0092699) for financial support. This work was also supported by a graduate research award to J.Z. from the Center for Nanotechnology at the University of Washington.

${ }^{1}$ L. Sun and R. M. Crooks, J. Am. Chem. Soc. 122, 12340 (2000).

${ }^{2}$ C. Liu, Y. Fan, H. T. Cong, H. M. Cheng, and M. S. Dreselhaus, Science 286, 1127 (1999).

${ }^{3}$ M. Zheng, A. Jagota, E. D. Semke, B. A. Diner, R. S. Mclean, S. R. Lustig, R. E. Richardson, and N. G. Tassi, Nature (London) 2, 338 (2003).

${ }^{4}$ D. W. Deamer and M. Akeson, Trends Biotechnol. 18, 147 (2000).

${ }^{5}$ P. M. Ajayan and S. Iijima, Nature (London) 361, 333 (1993).

${ }^{6}$ P. M. Ajayan, O. Stephan, P. Redlich, and C. Colliex, Nature (London) 375, 564 (1995)

${ }^{7}$ B. Roux and M. Karplus, Annu. Rev. Biophys. Biomol. Struct. 23, 731 (1994).

${ }^{8}$ D. Fu, A. Libson, L. J. W. Miercke, C. Weitzman, P. Nollert, J. Krucinski, and R. M. Stroud, Science 290, 481 (2000).
${ }^{9}$ S. A. Miller, V. Y. Young, and C. R. Martin, J. Am. Chem. Soc. 123, 12335 (2001).

${ }^{10}$ L. Sun and R. M. Crooks, J. Electrochem. Soc. 122, 12340 (2000).

${ }^{11}$ J. Li, C. Papadopoulos, and J. Xu, Nature (London) 402, 253 (1999).

${ }^{12}$ E. Dujardin, T. W. Ebbesen, H. Hiura, and K. Tanigaki, Science 265, 1850 (1994).

${ }^{13}$ T. W. Ebbesen, J. Phys. Chem. Solids 57, 951 (1996).

${ }^{14}$ O. Beckstein and M. S. P. Sansom, Proc. Natl. Acad. Sci. U.S.A. 100, 7063 (2003).

${ }^{15}$ O. Beckstein, P. C. Biggin, and M. S. P. Sansom, J. Phys. Chem. B 105, 12902 (2001).

${ }^{16}$ T. W. Allen, S. Kuyucak, and S. H. Chung, J. Chem. Phys. 111, 7985 (1999).

${ }^{17}$ G. Hummer, J. C. Rasaiah, and J. P. Noworyta, Nature (London) 8, 188 (2001).

${ }^{18}$ A. Kalra, S. Garde, and G. Hummer, Proc. Natl. Acad. Sci. U.S.A. 100, 10175 (2003).

${ }^{19}$ G. S. Heffelfinger and F. van Swol, J. Chem. Phys. 100, 7548 (1994); G. S. Heffelfinger and D. M. Ford, Mol. Phys. 94, 659 (1998); D. M. Ford and G. S. Heffelfinger, ibid. 94, 673 (1998); P. Thompson and G. S. Heffelfinger, J. Chem. Phys. 109, 6406 (1998); P. I. Pohl and G. S. Heffelfinger, J. Membr. Sci. 155, 1 (1999).

${ }^{20}$ J. M. D. MacElroy, J. Chem. Phys. 101, 5274 (1994); J. M. D. MacElroy and M. J. Boyle, Chem. Eng. J. 74, 85 (1999).

${ }^{21}$ R. F. Cracknell and D. Nicholson, J. Chem. Soc., Faraday Trans. 90, 1487 (1994); R. F. Cracknell, D. Nicholson, and N. Quirke, Phys. Rev. Lett. 74, 2463 (1995).

${ }^{22}$ Q. Zhang, J. Zheng, A. Shevade, L. Zhang, S. H. Gehrke, G. S. Heffelfinger, and S. Jiang, J. Chem. Phys. 117, 808 (2002).

${ }^{23}$ Z. Mao and S. B. Sinnott, J. Phys. Chem. B 105, 6916 (2001).

${ }^{24}$ A. I. Skoulidas, D. M. Ackerman J. K. Johnson, and D. S. Sholl, Phys. Rev. Lett. 89, 185901 (2002).

${ }^{25}$ E. A. Müller, L. F. Rull, L. F. Vega, and K. E. Gubbins, J. Phys. Chem. 100, 1189 (1996).

${ }^{26}$ T. Kyotani, S. Nakazaki, W. Xu, and A. Tomita, Carbon 39, 771 (2001).

${ }^{27}$ H. J. C. Berendsen, J. P. M. Postma, W. F. von Gunsteren, and J. Hermans, Intermolecular Forces (Reidel, Dordrecht, 1981).

${ }^{28}$ W. L. Jorgensen, J. M. Briggs, and M. L. Conteras, J. Phys. Chem. 94, 1683 (1990).

${ }^{29}$ M. Haughney, M. Ferrario, and I. R. McDonald, Mol. Phys. 58, 849 (1986).

${ }^{30}$ I. Brovchenko, A. Geiger, A. Oleinikova, and D. Paschek, Eur. Phys. J. E 12, 69 (2003).

${ }^{31}$ A. Waghe, J. C. Rasaiah, and G. Hummer, J. Chem. Phys. 117, 10789 (2002).

${ }^{32}$ J. Marti and M. C. Gordillo, Phys. Rev. E 64, 021504 (2001).

${ }^{33}$ D. J. Mann and M. D. Hall, Phys. Rev. Lett. 90, 195503 (2003).

${ }^{34}$ A. V. Shevade, S. Jiang, and K. E. Gubbins, J. Chem. Phys. 113, 6933 (2000).

${ }^{35}$ J. Sekulić, J. E. ten Elshof, and D. H. A. Blank, Langmuir 21, 508 (2005). 